\title{
Cascade Distiller System Performance Testing Interim Results
}

\author{
Michael R. Callahan ${ }^{1}$, Stuart Pensinger ${ }^{1}$, Miriam J. Sargusingh ${ }^{2}$ \\ NASA Johnson Space Center, Houston, TX, 77058
}

The Cascade Distillation System (CDS) is a rotary distillation system with potential for greater reliability and lower energy costs than existing distillation systems. Based upon the results of the 2009 distillation comparison test (DCT) and recommendations of the expert panel, the Advanced Exploration Systems (AES) Water Recovery Project (WRP) project advanced the technology by increasing reliability of the system through redesign of bearing assemblies and improved rotor dynamics. In addition, the project improved the CDS power efficiency by optimizing the thermoelectric heat pump (TeHP) and heat exchanger design. Testing at the NASA-JSC Advanced Exploration System Water Laboratory (AES Water Lab) using a prototype Cascade Distillation Subsystem (CDS) wastewater processor (Honeywell d International, Torrance, Calif.) with test support equipment and control system developed by Johnson Space Center was performed to evaluate performance of the system with the upgrades as compared to previous system performance. The system was challenged with Solution 1 from the NASA Exploration Life Support (ELS) distillation comparison testing performed in 2009. Solution 1 consisted of a mixed stream containing human-generated urine and humidity condensate. A secondary objective of this testing is to evaluate the performance of the CDS as compared to the state of the art Distillation Assembly (DA) used in the ISS Urine Processor Assembly (UPA). This was done by challenging the system with ISS analog waste streams. This paper details the results of the AES WRP CDS performance testing.

\footnotetext{
${ }^{1}$ Principle Investigator, Crew and Thermal Systems Division, 2101 NASA Parkway/EC3

${ }^{2}$ Systems Engineer, Crew and Thermal Systems Division, 2101 NASA Parkway/EC2
} 